CERN-PH-TH/2007-161

\title{
Composition of UHECR and the Pierre Auger Observatory Spectrum
}

\author{
Katsushi Arisaka ${ }^{a}$, Graciela B. Gelmini ${ }^{a, b}$, Matthew Healy ${ }^{a}$, \\ Oleg Kalashev ${ }^{c}$ and Joong Lee ${ }^{a}$ \\ ${ }^{a}$ Department of Physics and Astronomy, UCLA, Los Angeles, CA 90095-1547, USA \\ ${ }^{b}$ CERN, PH-TH, CH-1211 Genève 23, Switzerland \\ ${ }^{c}$ INR RAS, 60th October Anniversary pr. 7a, 117312 Moscow, Russia
}

\begin{abstract}
We fit the recently published Pierre Auger ultra-high energy cosmic ray spectrum assuming that either nucleons or nuclei are emitted at the sources. We consider the simplified cases of pure proton, or pure oxygen, or pure iron injection. We perform an exhaustive scan in the source evolution factor, the spectral index, the maximum energy of the source spectrum $Z \times E_{\max }$, and the minimum distance to the sources. We show that the Pierre Auger spectrum agrees with any of the source compositions we assumed. For iron, in particular, there are two distinct solutions with high and low $E_{\max }$ (e.g. $6.4 \times 10^{20} \mathrm{eV}$ and $2 \times 10^{19} \mathrm{eV}$ ) respectively which could be distinguished by either a large fraction or the near absence of proton primaries at the highest energies. We raise the possibility that an iron dominated injected flux may be in line with the latest composition measurement from the Pierre Auger Observatory where a hint of heavy element dominance is seen.
\end{abstract}

PACS numbers: 98.70.Sa 


\section{Introduction}

The Greisen-Zatsepin-Kuzmin (GZK) cutoff [1] at $4 \times 10^{19} \mathrm{eV}$ seems not to be present in the data of the AGASA ground array [2] but it appears in the data of the HiRes air fluorescence detector [3, 4]. This controversy can be addressed by the Pierre Auger Observatory [5], a hybrid combination of charged particle detectors and fluorescence telescopes, as it continues to accumulate data. We study here the most recent spectrum published by the Pierre Auger Observatory [6].

Using the surface array, the Pierre Auger Collaboration presented [6] an update to their previous result [7] that includes two additional years of data and an integrated aperture $\left(5165 \mathrm{~km}^{2} \mathrm{sr}\right.$ yr) nearly equivalent to that of the HiRes experiment. The updated spectrum begins at an energy of $2.5 \times 10^{18} \mathrm{eV}$, the energy at which the surface array becomes fully efficient within the zenith angle range 0-60 [8], and ends with a highest observed energy of $\sim 1.8 \times 10^{20} \mathrm{eV}$. Energies are determined in a simulation independent way assuming constant intensity and calibrating the ground observable S(1000) against the fluorescence detector energy for the subset of showers (known as golden hybrid showers) that contain reconstructions from both detectors. The method leads to a statistical error of $8 \%$ and a systematic error of $22 \%$ [6, 9] on the energy.

The origin of cosmic rays with energies beyond the GZK cutoff remain an outstanding open question in astroparticle physics and cosmology [2, 3, 4, 10]. Nucleons cannot be significantly deflected by the magnetic fields of our galaxy for energies above the "ankle", i.e. above $10^{18.5} \mathrm{eV}$. This and the absence of a correlation of arrival directions with the galactic plane indicate that, if nucleons are the primary particles of the ultra high energy cosmic rays (UHECR), these nucleons should be of extragalactic origin. Moreover, nucleons as well as photons with energies above $5 \times 10^{19} \mathrm{eV}$ could not reach Earth from a distance beyond 50 to $100 \mathrm{Mpc}$ [11, 12] thus sources should be found within this distance. Nucleons scatter off the cosmic microwave background (CMB) photons with a resonant photoproduction of pions $p \gamma \rightarrow \Delta^{*} \rightarrow N \pi$, where the pion carries away $\sim 20 \%$ of the original nucleon energy. Photons with comparable energy pair-produce electrons and positrons on the radio background.

Intervening sheets of large scale intense extra galactic magnetic fields (EGMF), with intensities $B \sim 0.1-1 \times 10^{-6} \mathrm{G}$, could provide sufficient angular deflection for protons to explain the lack of observed sources in the directions of arrival of UHECR. However, recent realistic simulations of the expected large scale EGMF show that strong deflections could only occur when particles cross galaxy clusters. Except in the regions close to the Virgo, Perseus and Coma clusters the magnetic fields are not larger than

$3 \times 10^{-11} \mathrm{G}[13$ and the deflections expected are not important (however see Ref. [14]).

Heavy nuclei are an interesting possibility for UHECR primaries, since they could be produced at the sources with larger maximum energies and would more easily be deflected by intervening magnetic fields. Both AGASA and HiRes data favor a dominance of light hadrons, consistent with being all protons, in the composition of UHECR above $10^{19} \mathrm{eV}$ [15]. These data are consistent with models in which all UHECR 
above $10^{18} \mathrm{eV}$ are due to extragalactic protons [16]. The Pierre Auger Observatory has presented an elongation rate that is better represented by a fit containing a break point in the slope at $2 \times 10^{18} \mathrm{eV}$. Below the break point the spectrum is consistent with a progressively lighter composition but above the break the composition is consistent with a constant and mixed composition up to the highest energies [17. This raises the possibility of a significant fraction of heavier elements in the range of the GZK cutoff.

Whether particles can be emitted with the necessary energies by astrophysical accelerators, such as active galactic nuclei, jets or extended lobes of radio galaxies, or even extended objects such as colliding galaxies and clusters of galaxies, is still an open question. The size and possible magnetic and electric fields of these astrophysical sites make it plausible for them to accelerate protons and nuclei to a maximum energy of $Z \times 10^{21} \mathrm{eV}$, where $Z$ is the number of protons in each nucleus. Larger emission energies would require a reconsideration of possible acceleration models or sites.

A galactic component of the UHECR flux, which could be important up to energies $10^{19} \mathrm{eV}$, should consist of heavy nuclei, given the lack of correlation with the galactic plane of events at this energy (outside the galactic plane galactic protons would be deflected by a maximum of $15-20^{\circ}$ at this energy [18]).

In this paper we fit the Pierre Auger UHECR spectrum above the energy $E_{\text {cut }}=$ $1 \times 10^{19} \mathrm{eV}$ (and for comparison we also use two other values of $E_{\text {cut }}, 2.5 \times 10^{18} \mathrm{eV}$ and $4 \times 10^{19} \mathrm{eV}$ ) assuming that either protons or nuclei are emitted at the sources. The UHECR spectrum predicted depends on the slope and maximum energy of the nucleon or nucleus spectrum emitted at the source, the distribution of sources, and the intervening backgrounds. We take a phenomenological approach in choosing the range of the several relevant parameters which determine the cosmic ray flux, namely we take for each of them a range of values mentioned in the literature, without attempting to assign them to particular sources or acceleration mechanisms. We consider the simplified case in which either only protons, or only oxygen nuclei, or only iron nuclei would be emitted by the sources. Although these are not realistic models for the injected composition, we expect to gain some understanding of how well a heavy or intermediate or light elements dominated composition in the injected spectrum can account for the observed spectrum.

The ankle in the UHECR spectrum at energies $10^{18} \mathrm{eV}-10^{19} \mathrm{eV}$ can be explained either by $e^{ \pm}$pair production by extragalactic protons interacting with the CMB [16] or by a change from one component of the UHECR spectrum to another. We take into account the first possibility by fitting the Pierre Auger spectrum above $2.5 \times 10^{18} \mathrm{eV}$ with a flux of protons emitted at the sources. This possibility can still be consistent with the proton-dominated composition observed by HiRes.

The second explanation of the ankle, in which the extragalactic component dominates at energies above the ankle, assumes the existence of a low energy component (LEC) when necessary to fit the UHECR spectrum at energies lower than 1 to $4 \times 10^{19} \mathrm{eV}$. This LEC can be dominated by galactic Fe or by a different population of lower energy extragalactic nucleons. Here we do not address the issue of what the LEC is. We only assume that, if it exists, it becomes negligible at energies above the energy $E_{\text {cut }}$ at which 
we start our fit, i.e. either $1 \times 10^{19} \mathrm{eV}$ or $4 \times 10^{19} \mathrm{eV}$. In this case we study both the case of protons as well as that of nuclei ( $\mathrm{Fe}$ or $\mathrm{O}$ ) emitted at the sources.

Our calculations do not take into account deflections. Since we assume typical extragalactic magnetic fields not larger than $3 \times 10^{-11} \mathrm{G}$ [13] outside large clusters, the deflections of iron nuclei become important for energies below $1 \times 10^{19} \mathrm{eV}$. Therefore we only consider nucleons below this energy.

When $E_{\text {cut }}>2.5 \times 10^{18} \mathrm{eV}$, besides fitting the spectrum above $E_{\text {cut }}$, we require that the spectrum we predict is never above the measured spectrum at energies between $2.5 \times 10^{18} \mathrm{eV}$ and $E_{\text {cut }}$.

The plan of the paper is the following. In Section II, we explain how we model the sources and the propagation of particles. In Section III, we show the goodness of fit of the many models we consider. In Section IV we show the average composition and the spectra of some of the models. We conclude in Section V.

\section{Modeling of the sources and particle propagation}

We use a numerical code originally described in Ref. [19] to compute the flux of GZK photons produced by a uniform distribution of sources emitting originally only protons or nuclei. The code uses the kinematic equation approach and calculates the propagation of nuclei, nucleons, stable leptons and photons using the standard dominant processes. This is the same numerical code as in Ref. [20], where the latest version of the code is described in detail.

UHE particles lose their energy in interactions with the electromagnetic background, which consists of CMB, radio, infra-red and optical (IRO) components, as well as EGMF. Protons are sensitive essentially to the CMB only, while for UHE photons and nuclei the radio and IRO components are respectively important, besides the CMB. Secondary photons are always subdominant and thus do not contribute significantly to the fits. Therefore the radio background assumed is not important. For the IRO background component we used the model of Ref. [21]. This background is important for the photodisintegration of nuclei and to transport the energy of secondary photons in the cascade process from the 0.1 - $100 \mathrm{TeV}$ energy range to the $0.1-100 \mathrm{GeV}$ energy range observed by EGRET, and the resulting flux in this energy range is not sensitive to details of the IRO background models. The possible deflection due to extragalactic magnetic fields is not included in the calculations. These deflections could considerably extend the path of heavy nuclei below $1 \times 10^{19} \mathrm{eV}$, but we do not consider the propagation of nuclei at these energies.

Notice that if neutrons are produced at the sources, the results at high energies are very close to those obtained with protons. The interactions of neutrons and protons with the intervening backgrounds are almost identical and when a neutron decays practically

all of its energy goes to the final proton (while the electron and neutrino are produced with energies $10^{17} \mathrm{eV}$ or lower).

As is usual, we take the spectrum of an individual UHECR source to be of the 
form:

$$
F(E)=f E^{-\alpha} \Theta\left(Z E_{\max }-E\right),
$$

where $f$ provides the flux normalization, $\alpha$ is the spectral index and $E_{\max }\left(Z E_{\max }\right)$ is the maximum energy to which protons (or nuclei with charge $Z$ ) can be accelerated at the source.

We are implicitly assuming that the sources are astrophysical, since these are the only ones which could produce solely protons (or neutrons) and nuclei as UHECR primaries. Astrophysical acceleration mechanisms often result in $\alpha \gtrsim 2$ [22], however, harder spectra, $\alpha \lesssim 1.5$ are also possible, see e.g. Ref. [23]. In reality, the spectrum may differ from a power-law, it may even have a peak at high energies [24]. AGN cores could accelerate protons with induced electric fields, similar to what happens in a linear accelerator, and this mechanism would produce an almost monoenergetic proton flux, with energies as high as $10^{20} \mathrm{eV}$ or higher [25]. Here, we consider the power law index to be in the range $1 \leq \alpha \leq 2.7$. An injected proton spectrum with $\alpha \geq 2.5$ does not require an extra contribution to fit the UHECR data, except at very low energies $E<10^{18} \mathrm{eV}$ [26]. For $\alpha \leq 2$ an extra low energy component (LEC) is required to fit the UHECR data at $E<1 \times 10^{19} \mathrm{eV}$. Here we will consider values of $E_{\max }$ up to $10^{21} \mathrm{eV}$.

We assume a standard cosmological model with a Hubble constant $H=$ $70 \mathrm{~km} \mathrm{~s}^{-1} \mathrm{Mpc}^{-1}$, a dark energy density (in units of the critical density) $\Omega_{\Lambda}=0.7$ and a dark matter density $\Omega_{\mathrm{m}}=0.3$. The total source density in this model can be defined by

$$
n(z)=n_{0}(1+z)^{3+m} \Theta\left(z_{\max }-z\right) \Theta\left(z-z_{\min }\right),
$$

where $m$ parameterizes the source density evolution, in such a way that $m=0$ corresponds to non-evolving sources with constant density per comoving volume, and $z_{\min }$ and $z_{\max }$ are respectively the redshifts of the closest and most distant sources.

The energy of the background photons increases linearly with $(z+1)$ thus the GZK energy, about $3 \times 10^{19} \mathrm{eV}$ at $z=0$, decreases as $1 /(z+1)$ at redshift $z$. Moreover, the particles produced with that energy at redshift $\mathrm{z}$ will arrive to us with energy redshifted as $1 /(z+1)$, namely with characteristic energy $E=3 \times 10^{19} \mathrm{eV} /(z+1)^{2}$. This means that for $z>1, E<(3 / 4) \times 10^{19} \mathrm{eV}$, and for $z>2, E<(3 / 9) \times 10^{19} \mathrm{eV}$. We conclude that sources with $z>1$ have a negligible contribution to the UHECR flux above $1 \times 10^{19} \mathrm{eV}$ and those with $z>2$ do not contribute above $3 \times 10^{18} \mathrm{eV}$. Thus any value of $z_{\max } \geq 1$ or 2 , respectively would give the same results.

We have considered several possible values of $m$, i.e. $m=4,2,0,-2$ in this paper. The fast and slow star formation rate evolution models of Ref. [21] have $m=4$ and $m=3$ respectively at $z<1$ (and become constant close to $z=1$ up to $z>5$ ). The evolution of radio galaxies and AGNs [27, is somewhat faster than $m=3$ below $z=2$ (reaches a maximum at a $z$ between 2 and 3 and then decreases-see Fig. 6 of [28]). Smaller positive values of $m$ up to $m=0$, correspond to an older star population evolution and is taken here as a lower limit to the value of $m$ at low redshifts for protons. Negative values of $m$ have been mentioned in the literature only for very massive clusters, which only formed 
recently. However, accretion shocks in clusters might accelerate heavy nuclei but not protons to the energies necessary to account for the ultrahigh energy cosmic rays [29].

The value of $z_{\min }$ is connected to the density of sources. Quite often in the literature the minimal distance to the sources is assumed to be negligible (i.e. comparable to the interaction length). We also consider non-zero minimum distances of up to $50 \mathrm{Mpc}$ $\left(z_{\min }=0.01\right)$, as inferred from the small-scale clustering of events seen in the AGASA data [30]. Contrary to AGASA, HiRes does not see a clustering component in its own data [31]. The combined dataset shows that clustering still exists, but it is not as significant as in the data of AGASA alone [32. Note, that the non-observation of clustering in the HiRes stereo data does not contradict the result of AGASA, because of the small number of events in the sample [33. Assuming proton primaries and a small EGMF (following Ref. [13]), it is possible to infer the density of the sources [33, 34] from the clustering component of UHECR. AGASA data alone suggest a source density of $2 \times 10^{-5} \mathrm{Mpc}^{-3}$, which makes plausible the existence of one source within $25 \mathrm{Mpc}$ of us. However, the HiRes negative result on clustering requires a larger density of sources and, as a result, a smaller distance to the nearest one of them. Larger values of the EGMF (as found in Ref. [14]), and/or some fraction of iron in the UHECR, have the effect of reducing the required number of sources and, consequently, increasing the expected distance to the nearest one.

Most of the energy in GZK photons cascades down to below the pair production threshold for photons on the CMB and infrared backgrounds. In general, for $\alpha<2$ the diffuse extragalactic gamma-ray flux measured by EGRET [35] at $\mathrm{GeV}$ energies may impose a constraint on the GZK photon flux at high energies, which we take into account and found not relevant for any of the models we study here.

\section{Goodness of fit of different source models}

In this section we estimate the flux predicted by the models by fitting the Pierre Auger UHECR spectrum. We proceed using the method explained in Ref. [36].

We fit the Pierre Auger UHECR data assuming many different injected spectra. We assume an injected spectrum given by Eq. 1, a uniform distribution of sources with a density as in Eq. 2 with $z_{\max }=3$ and, $z_{\min }=0$ or 0.005 or 0.01 and $m=4$ or 2 or 0 or -2 . We consider then many different spectra resulting from changing the slope $\alpha$ and the maximum energy $E_{\max }$ in Eq. 1 within the ranges $1 \leq \alpha \leq 2.7$ and $10^{19} \mathrm{eV} \leq E_{\max } \leq 1.28 \times 10^{21} \mathrm{eV}$ in steps $\alpha_{n}=1+0.1 n$, with $n=0$ to 17 and $E_{\ell}=1 \times 10^{19} \mathrm{eV} \times 2^{\ell}$, with $\ell=0$ to 7 . For each one of the models so obtained we compute the predicted UHECR spectrum arriving to us from all sources.

In order to compare the predicted flux with the data, we also take into account the experimental error in the energy determination as proposed in Ref. [37]. We take a lognormal distribution for the error in the energy reconstructed by the experiment with respect to the true value of energy of the UHECR coming into the atmosphere. To find the expected flux we convolute the spectrum predicted by each model with 
the lognormal distribution in energy with the width given by the Pierre Auger energy error $\Delta E / E=8 \%$ [9] (the parameter $\sigma$ in Eq. (5) of Ref. [37], the standard deviation of $\log _{10} E$, is $\left.\sigma=(\Delta E / E) / \ln (8) \simeq(\Delta E / E) / 2.08\right)$. This procedure results in small but nonnegligible changes in the predicted spectra which are then compared to the observed spectrum. In particular, there are events predicted with an energy larger than the maximum injected energy $Z E_{\max }$. Somewhat arbitrarily we consider the energy beyond which no event is predicted to be $(1+10 \Delta E / E) Z E_{\max }$. Moreover, we take into account that there is about a factor of 2 between the energy of a photon event and the energy measured if the event is reconstructed assuming it is a proton [38]. Thus we divide the energy of the predicted GZK photon energy by 2 before comparing it with the observed Pierre Auger spectrum. However, the GZK photons are always subdominant in the flux of UHECR [20, 36] thus they do not affect the goodness of the fits (and at present the GZK photon fractions are not constrained by Auger upper bounds- see Fig. 18 of Ref. [39]).

With each predicted spectrum we fit the UHECR data from $E_{\text {cut }}$ up to a bin past the last published bin of the spectrum (which is the $10^{20.3} \mathrm{eV}$ bin of the Pierre Auger Observatory). The extra bin extends from the maximum experimental point of the observed spectrum, $10^{20.4} \mathrm{eV}$ [6] (which is also empty) to $(1+10 \Delta E / E) Z E_{\max }$ (where $Z E_{\max }$ is the maximum energy assumed for the injected spectrum in Eq. 1). We do this because the assumed injection spectrum could produce an event in this bin even though the experiment did not observe one. If the maximum possible energy, $(1+10 \Delta E / E) Z E_{\max }$, is less than the maximum bin of the published spectrum the additional bin is not needed and therefore not added. In certain assumed injection spectra the maximum possible energy is less than the energy of the most energetic event observed. In this case the assumption is not valid on the face of it and therefore immediately disqualified. Situations like this can be seen in Fig. 1 and Fig. 2 as the empty regions.

We also change $E_{\text {cut }}$ and fit the UHECR data from $2.5 \times 10^{18} \mathrm{eV}$ (only with injected protons) or $4 \times 10^{19} \mathrm{eV}$ and compare the results with those of $1 \times 10^{19} \mathrm{eV}$. We show how this affects the goodness of the fit in Fig. 5 using proton sources.

The expected number of events in each bin between $E_{\text {cut }}$ and the maximum energy bin is computed using the exposure of the Pierre Auger Observatory, $5165 \mathrm{~km}^{2}$ sr yr [6]. The aperture remains constant with increasing energy.

Fitting the UHECR data with a predicted spectrum follows a procedure similar to that of Ref. [40] applied to the bins just mentioned. We compare the observed number of events in each bin with the number of events predicted by the models and choose the value of the parameter $f$ in Eq. 1, i.e. the amplitude of the injected spectrum, by maximizing the Poisson likelihood function. This is equivalent to minimizing $-2 \ln \lambda$, (i.e. the negative of the log likelihood ratio) [41]. This procedure amounts to choosing the value of $f$ so that the mean total number of events predicted (i.e. the sum of the average predicted number of events in all fitted bins) is equal to the total number of events observed. We then compute, using a Monte Carlo technique, the goodness of 
the fit, or $p$-value of the distribution, defined as the mean fraction of hypothetical experiments (observed spectra) with the same fixed total number of events which would result in a worse, i.e. smaller, Poisson likelihood than the one obtained (in the maximization procedure that fixed $f$ ). These hypothetical experiments are chosen at random according to the multinomial distribution of the model (with $f$ fixed as described). We have checked that this procedure when applied to bins with a large number of events gives the same result as a Pearson's $\chi^{2}$ fit, both for the value of the normalization parameter $f$ and for the goodness of fit. A higher $p$-value corresponds to a better fit since a greater number of hypothetical experimental results would yield a fit worse than the one we obtained.

We make one additional requirement on the fit to insure the predicted flux does not exceed the observed flux at energies below $E_{\text {cut }}$ and above $2.5 \times 10^{18} \mathrm{eV}$, the lowest energy of the published Auger spectrum. When $E_{\text {cut }}>2.5 \times 10^{18} \mathrm{eV}$, for each assumed spectrum (with $f$ fixed as described above) we calculate the $\chi^{2}$ for the data at energies below $E_{\text {cut }}$ using only the data points in which the predicted flux is above the observed flux (i.e. we take as zero the contribution to the $\chi^{2}$ of each data point for which the predicted flux is below the observed flux). We then require the $p$-value of the $\chi^{2}$ so obtained to be larger than 0.05. This constraint eliminates many combinations of $\alpha$ and $E_{\max }$ values. The regions eliminated by this requirement are the cross hatched regions in Fig. 1, 2, 4 and 5. This low energy constraint would, however, be too restrictive if somehow the extragalactic cosmic rays below some threshold energy between $2.5 \times 10^{18}$ $\mathrm{eV}$ and $E_{\text {cut }}$ do not reach Earth - for example, due to magnetic confinement at the source. In this case, the deficit of extragalactic flux below the threshold energy should be made up by a (possibly galactic) LEC.

Fig. 1 and 2 show in a logarithmic scale the color coded $p$-value of the maximum Poisson likelihood value obtained for each model as a function of $E_{\max }$ and $\alpha$, for $m=4$ and $m=0$, respectively. The top, middle and lower panels correspond to proton, oxygen, and iron emitted by the sources, respectively, while the columns from left to right correspond to $z_{\text {min }}=0,0.005,0.01$, respectively. Overall, the cross hatched region (in which the flux predicted at energies $2.5 \times 10^{18} \mathrm{eV}<E<E_{\text {cut }}$ exceeds the observed one) includes many regions of $E_{\max }, \alpha$ which would otherwise provide good fits (red and orange regions where $p$-value $\geq 0.05$ ). In some instances the acceptable models lie just outside a cross hatched region and in some others no acceptable models remain. The blue, green-blue, or yellow regions do not provide good fits (if we choose only $p$-value $\geq 0.05$ to be acceptable).

When $m=4$, good models with pure proton injection have $\alpha=2.2$ and $E_{\max }=10^{20.2}-10^{21.1} \mathrm{eV}$ if $z_{\min }=0.000$ or $E_{\max }=10^{20.5}-10^{21.1} \mathrm{eV}$ if $z_{\min }=0.005$. If all sources are further than $50 \mathrm{Mpc}\left(z_{\min }=0.010\right)$ there are no good fits with proton injection, because the GZK cutoff becomes too sharp so the flux is too low at $E \leq 10^{20}$ $\mathrm{eV}$. As $m$ decreases there are relatively more sources near by, thus the initial energies are less redshifted and the sources contribute less to the spectrum at lower energies. This change is compensated in the models providing good fits by an increase in $\alpha$ (a 

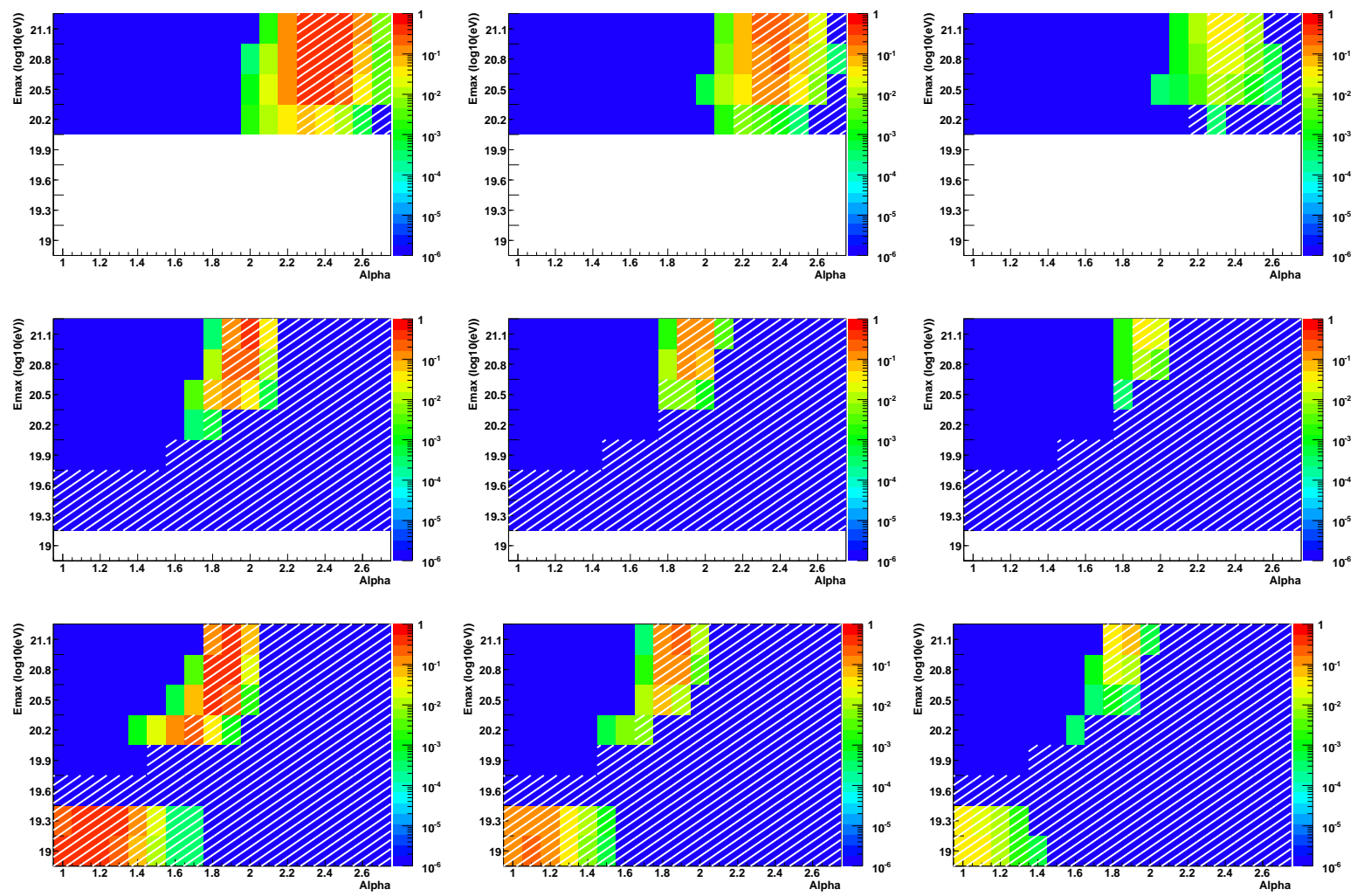

Figure 1. Color coded $p$-value plots as function of $E_{\max }$ and $\alpha$ for $E_{\text {cut }}=1 \times 10^{19} \mathrm{eV}$ and $m=4$, for $\mathrm{p}, \mathrm{O}$, or Fe emitted at the sources (top to bottom) and $z_{\min }=0,0.005$, 0.01 (left to right). White regions for $\mathrm{p}$ are eliminated because of energetic reasons. Cross hatched regions eliminated by the requirement at $2.5 \times 10^{18} \mathrm{eV}<E<E_{\text {cut }}$ (see text). Only orange and red regions have $p>0.05$.

steeper initial spectrum). However this leads to too large a flux at energies below $E_{\text {cut }}$ and the models are rejected by the low energy constraint. For $m=0$, for example, we see in Fig. 2 that a good fit region allowed by the low energy constraint exists only for $\alpha=2.4$ and $E_{\max }=10^{21.1} \mathrm{eV}$ and $z_{\min }=0$. For large $E_{\max }$ the proton accumulation below the GZK energy increases, thus the normalization of the predicted flux need to be lower to provide a good fit, this also lowers the flux predicted at low energies and the model is accepted by the low energy constraint. For lower values of $\alpha$ the $p$-values of the pure proton injection models are low because the predicted flux becomes too low at low energies, above but close to $E_{\text {cut }}=1 \times 10^{19} \mathrm{eV}$. This conclusion could be avoided if there was a non-negligible contribution from the LEC still contributing to the spectrum at energies above $1 \times 10^{19} \mathrm{eV}$.

No model with pure oxygen injection provides a good fit (outside the cross hatched region) with $m=4$ because for those models allowed by the low energy constraint, the protons resulting from the spallation of the $\mathrm{O}$ nuclei produce a too large bump in the predicted spectrum at low energies above but close to $E_{\text {cut }}$. However, for $m=0$ a high $E_{\max }$ region of good fits is present for pure O injected, with $\alpha=2.0, E_{\max }>10^{20.5} \mathrm{eV}$ if $z_{\min }=0$ or 0.05 , which disappears for larger distance to the sources. 

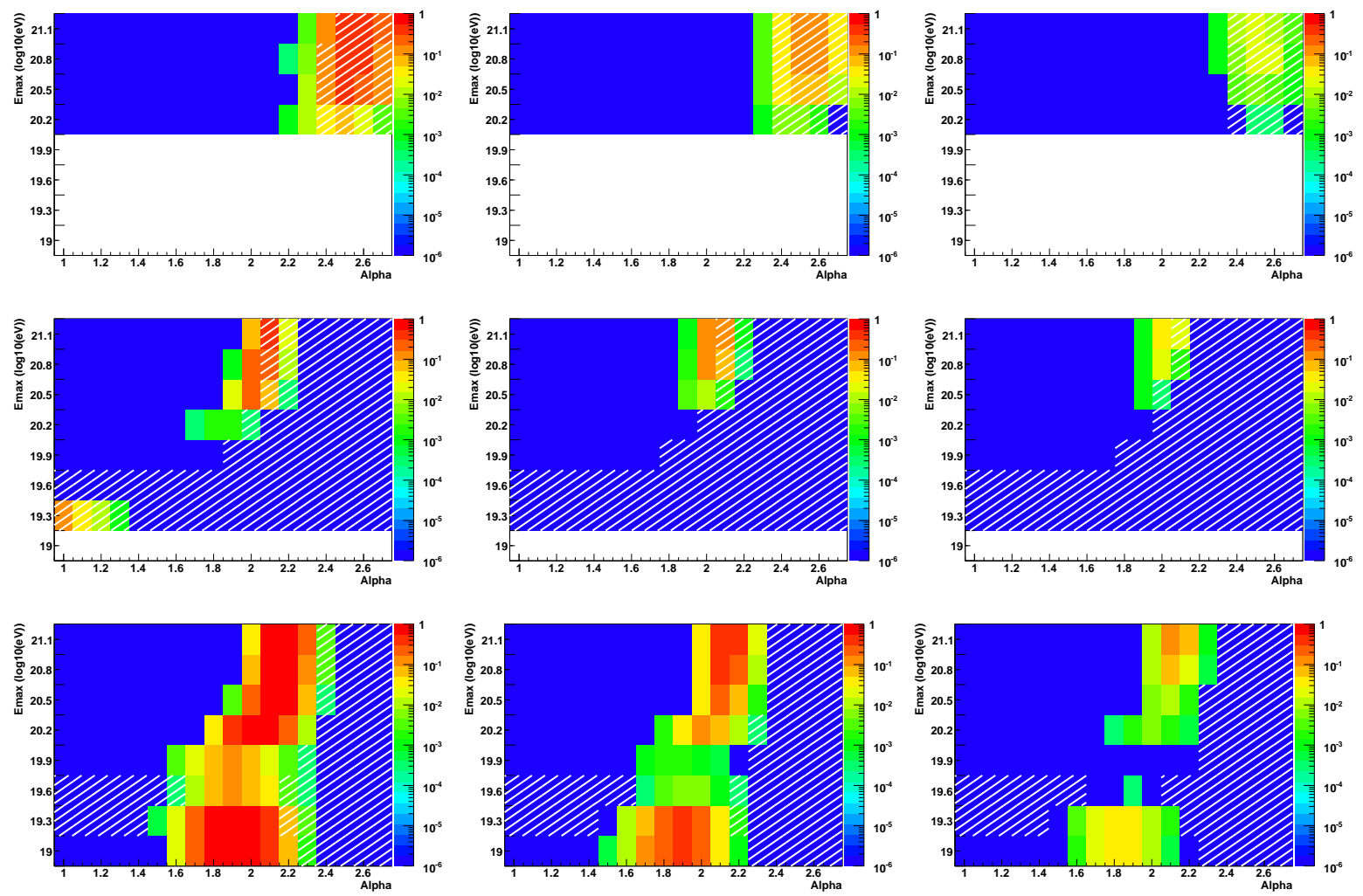

Figure 2. Same as Fig. [1 but for $m=0$. Notice that the best fit regions have shifted to higher values of $\alpha$.

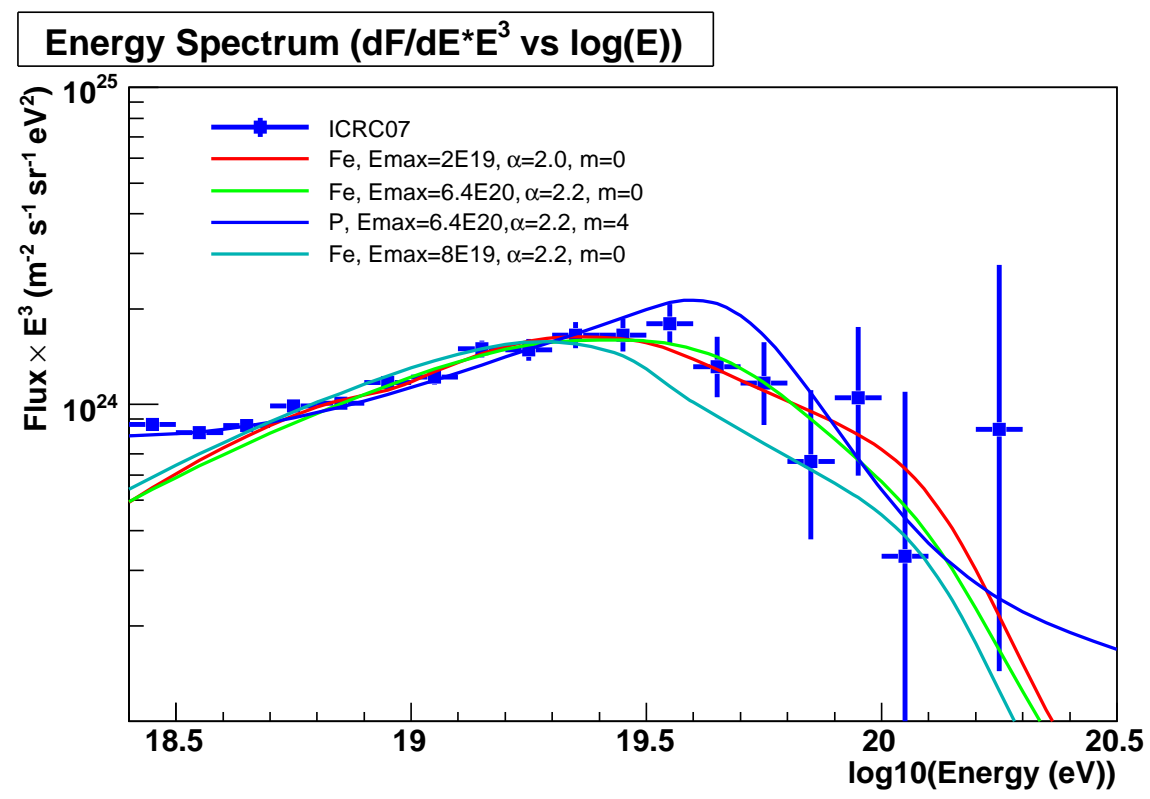

Figure 3. Examples of predicted UHECR spectra compared to the Auger data for several models providing good fits for p (blue), low $E_{\max } \mathrm{Fe}$ (red) and high $E_{\max } \mathrm{Fe}$ injected (green) and a bad fit (the intermediate $E_{\max }=8 \times 10^{19} \mathrm{eV}, \alpha=2.0, m=0$ Fe case, in teal). The respective $p$-values of these models are: $0.119,0.816,0.744$ and 0.0025 . Recall that the maximum energy is $Z E_{\max }$. 

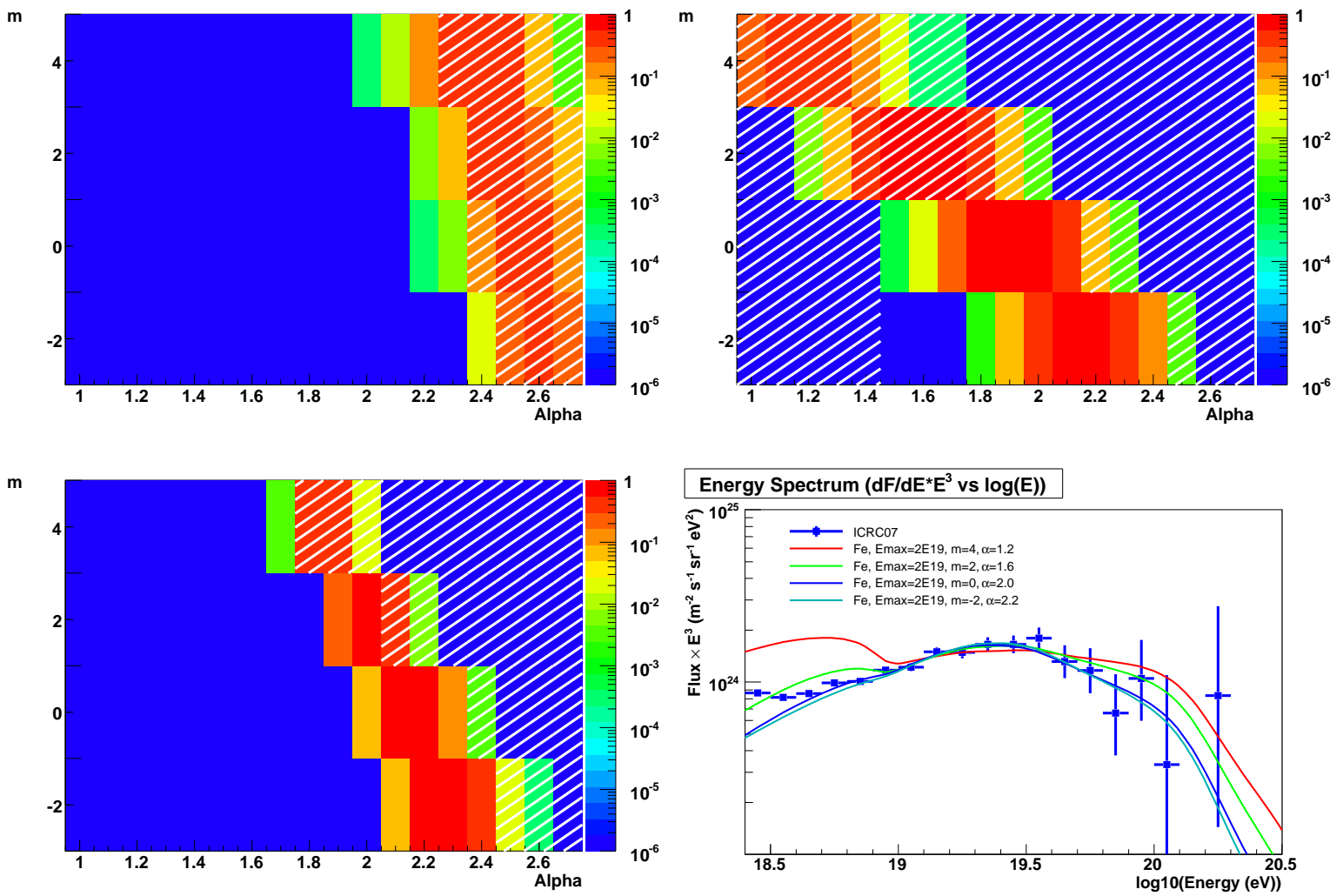

Figure 4. Degeneracy in $m$ and $\alpha$ for p injection with $E_{\max }=6.4 \times 10^{20} \mathrm{eV}$ (top left), Fe injection with maximum energy $Z E_{\max }=26 \times 2 \times 10^{19} \mathrm{eV}$ (top right) and $26 \times 6.4 \times 10^{20} \mathrm{eV}$ (bottom left). Predicted spectra for best fit cases as function of $m$ for Fe injection with $E_{\max }=2 \times 10^{19} \mathrm{eV}$ (bottom right). The $p$-values of the models listed are $0.458(m=4), 0.855(m=2), 0.816(m=0)$ and $0.828(m=-2)$.

Again for $m=4$, pure iron injection only provides acceptable models if the sources are close by, i.e. $z_{\min }=0$ if $\alpha=1.5-1.6$, and $E_{\max }=10^{20.2}-10^{20.5} \mathrm{eV}$. In contrast, $m=0$ leads to a larger region of satisfactory fits for iron injection which for $z_{\min }=0$ runs across all the range of $E_{\max }$ with $\alpha$ from 1.7 to 2.2. As the minimum distance to the sources increases to $25 \mathrm{Mpc}\left(z_{\min }=0.05\right)$ two separate regions of good fits remain for $\mathrm{Fe}$ injection: one at high $E_{\max }$ and $\alpha=1.9-2.2$ and one at low $E_{\max }$ with $\alpha=1.7-2.0$. As the minimum distance to the sources increases to $50 \mathrm{Mpc}\left(z_{\min }=0.1\right)$ the low $E_{\max }$ region of good fits disappears and the high $E_{\max }$ region shrinks to a single combination of parameters, $E_{\max }=10^{21.1} \mathrm{eV}, \alpha=2.0$ (and it is clear that no good fit would remain if the sources would be even further away). Recall that the actual maximum energy of nuclei is $Z E_{\max }$.

Closer sources, $z_{\min }=0$, always provide better fits, irrespective of the $m$ value, thus in the following figures (Fig. (3 to 66) $z_{\min }$ is set to zero. A few examples of the predicted spectra of models which provide good fits (i.e. having $p \geq 0.05$ ) or bad fits are shown

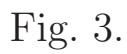


In Fig. 4 the degeneracy in $m$ and $\alpha$ is shown for three of the models providing good fits, p injected with $E_{\max }=6.4 \times 10^{20} \mathrm{eV}$ (top left), Fe injected with maximum energy $Z E_{\max }$ either $26 \times 2 \times 10^{19} \mathrm{eV}$ (top right) or $26 \times 6.4 \times 10^{20} \mathrm{eV}$ (bottom left). The figure shows clearly that decreasing $m$ while increasing $\alpha$ yields the same results. The bottom right panel shows the predicted spectra for best fit cases as function of $m$ from -2 to 4 , for Fe injection with $E_{\max }=2 \times 10^{19} \mathrm{eV}$.

As we clearly see, requiring that the predicted flux does not exceed the observed flux below $E_{\text {cut }}$ and above $2.5 \times 10^{18} \mathrm{eV}$ (the hatched regions in Figs. 1 to 5 ) excludes a large number of otherwise good fits. Thus, the caveat we mentioned earlier against this constraint is relevant: the constraint would not hold if the extragalactic cosmic rays with energy below some threshold energy between $2.5 \times 10^{18} \mathrm{eV}$ and $E_{\text {cut }}$ somehow do not reach Earth (are not emitted at the sources).

The best fits for proton injection happen for larger values of $\alpha$ (steeper spectrum) in comparison to the best fits for iron injection. As mentioned above, the steeper spectrum for $\mathrm{p}$ injection results in excess flux at low energies, whereas the harder spectrum for Fe injection tends to give a deficit of flux at low energies. As $m$ decreases (there are relatively more sources nearby), in order to get a good fit $\alpha$ must increase to compensate having less particles at low energy (close but above $E_{\text {cut }}$ ) which means excess flux at energies $E<E_{\text {cut }}$. This means that only large values of $m$ give acceptable solutions for proton.

The best fits for iron and oxygen, on the other hand, are forbidden by the low energy constraint due to an excess of flux at $E<E_{\text {cut }}=1 \times 10^{19} \mathrm{eV}$ due to a bump consisting of protons produced by photodisintegration (see the red Fe spectrum example for $m=4$ in the bottom right panel of Fig. 4). For larger $m$ values, good fits require smaller $\alpha$ values which result in a larger flux at the higher energies which also means more photodisintegrated protons.

So far we have fitted the data above $E_{\text {cut }}=1 \times 10^{19} \mathrm{eV}$. In Fig. 5 we explore the changes in the fits due to different choices of $E_{\text {cut }}$, namely $2.5 \times 10^{18} \mathrm{eV}$ and $4 \times 10^{19} \mathrm{eV}$ besides $1 \times 10^{19} \mathrm{eV}$ for the case of proton sources with $m=4$. As mentioned earlier, each $E_{\text {cut }}$ is appropriate for different hypotheses for the energy at which the transition to extra-galactic sources occurs. The effect of $E_{\text {cut }}$ on the goodness of fit is shown in Fig. 5: the regions with acceptable $p$-values increase progressively with increasing $E_{\text {cut }}$. This is easily understood, since there are more events per bin at low energies, thus the error bars are smaller and fewer models provide a good fit for lower $E_{\text {cut }}$.

For $E_{\text {cut }}=2.5 \times 10^{18} \mathrm{eV}$, the point $\alpha=2.2, E_{\max }=10^{20.5} \mathrm{eV}$ provides the best fit although with $p<0.05$. If the first data bin, at the $10^{18.4} \mathrm{eV}$, is eliminated from the fit, the $p$-value becomes larger than 0.05. This is because the models with non-negligible $p$-value for this low $E_{\text {cut }}$ have a deficit of flux at the $10^{18.4} \mathrm{eV}$ bin, the bin which has the smallest error bar. So presumably, if an LEC is added to match the flux exactly at that first bin, their low goodness of fit could be improved.

Fitting the spectrum only above $4 \times 10^{19} \mathrm{eV}$, on the other hand, is easier and models with a wide range of $\alpha$ and $E_{\max }$ values provide good fits, especially for small values 

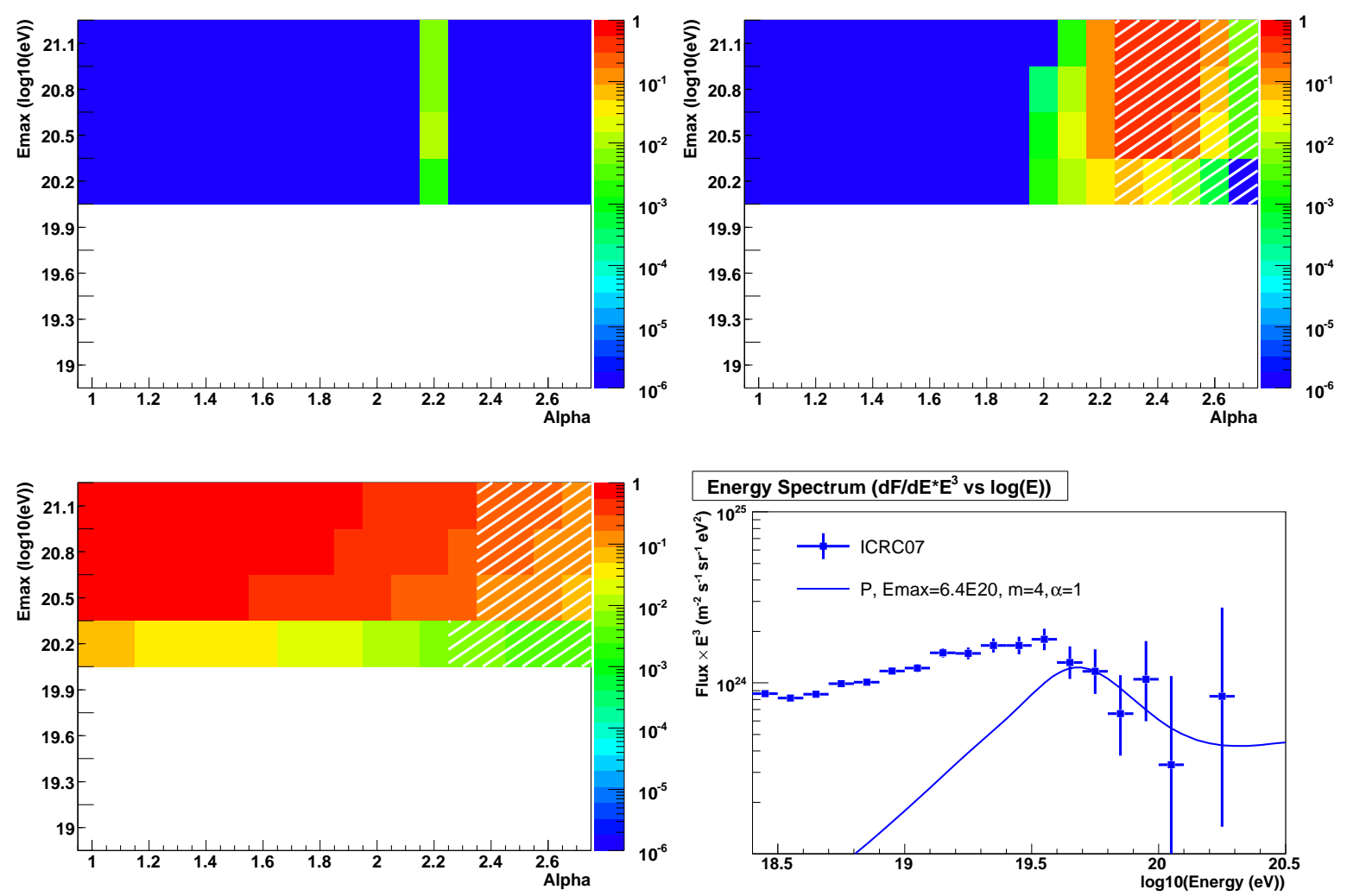

Figure 5. Color coded $p$-value plots for $z_{\min }=0$ and $m=4$ and only protons injected for different $E_{\text {cut }}$ values: $2.5 \times 10^{18} \mathrm{eV}$ (top left), $1 \times 10^{19} \mathrm{eV}$ (top right), and $4 \times 10^{19} \mathrm{eV}$ $E_{\text {cut }}$ (bottom left). Example of the predicted flux for $E_{\text {cut }}=4 \times 10^{19} \mathrm{eV}$ with $\alpha=1$, $E_{\max }=6.4 \times 10^{20} \mathrm{eV}$ (bottom right), model with $p$-value 0.809 .

of $\alpha$. Obviously these models require an LEC that makes up for the deficit in the flux below $4 \times 10^{19} \mathrm{eV}$. Even models with $\alpha=1$, a very flat spectrum, provide good fits. With such a hard injection spectrum the flux below $4 \times 10^{19} \mathrm{eV}$ is well under that of the Pierre Auger Observatory, as shown in the bottom right plot of Fig. 5. We can see from Fig. [ that if the LEC is assumed to extend all the way to $4 \times 10^{19} \mathrm{eV}$, almost any combination of parameters is satisfactory.

Although Fig. 5 only shows the case of proton injection with $m=4$, the same general consideration apply to nuclei and other values of $m$ as well (although, as we mentioned earlier, we cannot extend the fit all the way down to $2.5 \times 10^{18} \mathrm{eV}$ for nuclei since we do not take magnetic deflections into account). In the following we use $E_{\text {cut }}=1 \times 10^{19} \mathrm{eV}$.

\section{Composition and predicted spectra}

The two extreme best fit cases for iron with maximum energy $Z E_{\max }=26 \times 2 \times 10^{19} \mathrm{eV}$ and $Z E_{\max }=26 \times 6.4 \times 10^{20} \mathrm{eV}$ have interesting implications for the composition of the UHECR. As explained above, if $m=4$ only the high $E_{\max }$ case provides good fits to 

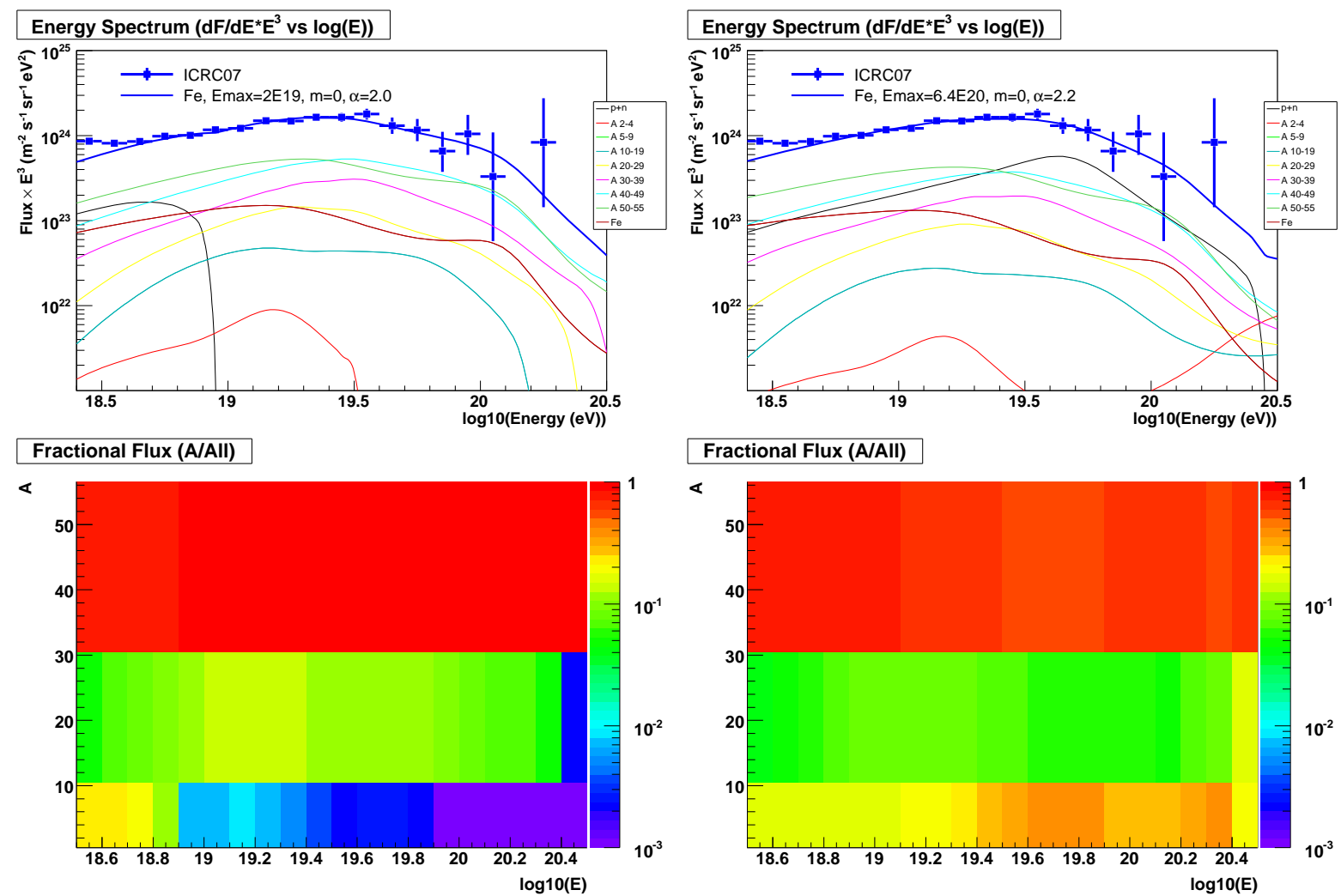

Fractional Flux (A/All)
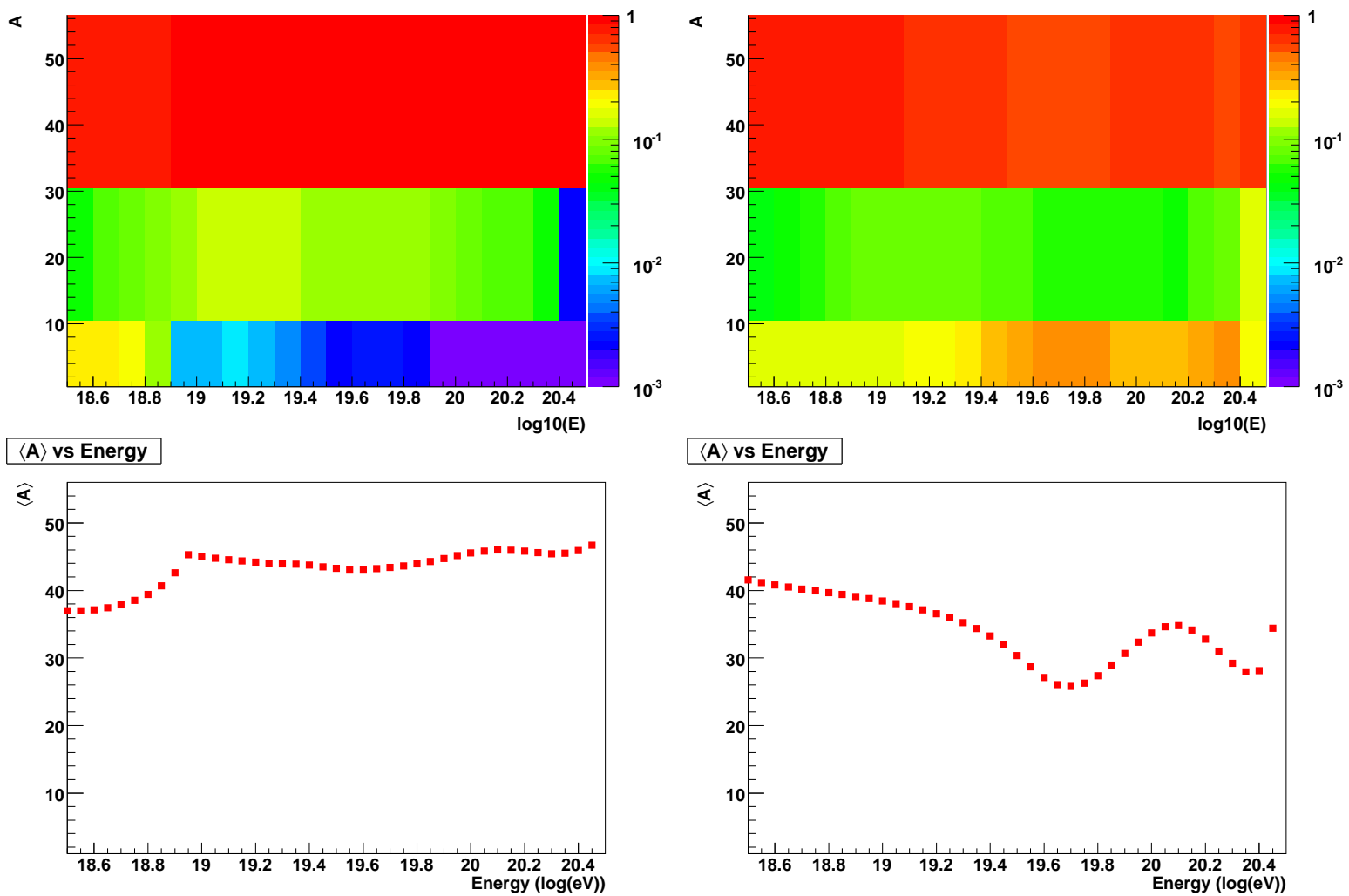

Figure 6. Detailed breakdown of the UHECR composition from photodisintegration of pure Fe injected for $Z E_{\max }=26 \times 2 \times 10^{19} \mathrm{eV}$ (left column) and $Z E_{\max }=$ $26 \times 6.4 \times 10^{20} \mathrm{eV}$ (right column) with $m=0$ ( $\alpha=2.0$ and 2.2 respectively). Comparison of the flux of different nuclei groups with the Pierre Auger spectrum (top panel) - the black line is the flux of photodisintegrated protons. Fractional flux for different nucleus species, divided into 3 groups according to their atomic number A: from 1 to 10, from 11 to 30, and from 30 to 56, color coded according to the fractional flux (middle panels). The flux is dominated by moderately heavy to heavy nuclei as indicated by the red regions, but for $Z E_{\max }=26 \times 6.4 \times 10^{20} \mathrm{eV}$ it still contains a significant proton flux (middle right). The light mass flux $(\mathrm{A}=1-10)$ is almost all protons and constitutes about $40 \%$ of the total. The average mass as function of energy shown in the bottom panels. 
the spectrum (and only if the sources are not farther away than $25 \mathrm{Mpc}$ ). Iron spallates during propagation and the breakdown of the resulting composition is shown in Fig. 6. The low $E_{\max }$ case is dominated by heavy elements with almost a total absence of primaries lighter than Boron (atomic number five) throughout the entire energy range. The proton flux from photodisintegration, the endpoint of which is $1 / 56$ of $Z E_{\max }$, ends below $10^{19} \mathrm{eV}$. The high $E_{\max }$ case does contain a significant proton fraction even up to the highest energies. The average mass of the composition for the low $E_{\max }$ case is upwards of $40 \mathrm{amu}$ (see bottom left in Fig. 6) nearing a flux of pure iron above an energy of $1 \times 10^{19} \mathrm{eV}$. The high $E_{\max }$ case has an average mass that varies with a minimum of $30 \mathrm{amu}$ (see bottom right in Fig. 6). The detailed breakdown of the compositional makeup of the UHECR flux is the key to distinguishing the two cases (see the two middle panels of Fig. 6 where the fraction of light, intermediate and heavy nuclei is represented in a color coded logarithmic scale). Indeed, the composition is dominated by the moderately heavy to heavy nuclei in both cases, but the fraction of protons for the high $\mathrm{E}_{\max }$ case is significant and can be upwards of $40 \%$ (notice the orange line at $\mathrm{A}=1$ to 10 for all energies in the middle right plot).

The latest data seem to indicate that the average $\mathrm{X}_{\max }$, which is characteristic of a flux dominated by light elements below $2 \times 10^{18} \mathrm{eV}$, transitions to a value that reflects heavier primaries especially above $\sim 2 \times 10^{19} \mathrm{eV}$ [17]. If this turns out to be true, it will be important to identify proton shower candidates in the high energy regime.

The two types of Fe injection solutions providing a good fit to the Pierre Auger spectrum were also found, with a different statistical analysis and modeling of predicted spectrum, in a very recent paper (see Ref. [42]) in which also the $\mathrm{X}_{\max }$ data of Auger are used (they seem to fix $m=0$ and $z_{\text {min }}=0$ ).

Very likely the UHECR sources will accelerate a mixed composition rather than all iron or oxygen or protons. We can think of a scenario, then, where the endpoint of each nucleus species is $Z \times 10^{19} \mathrm{eV}$ at the source where $Z$ is the charge of the nucleus. Then, the proton injection would end at a low energy, at 1/26 the maximum iron energy, and the maximum energy for heavy nuclei would not be so high as to result in too many protons from photodisintegration of heavy nuclei. In this scenario, unless the fraction of Fe (and other heavy elements) injected is very small, we can have a mixed composition spectrum that is dominated by heavy elements at the highest energies. If, on the contrary, the endpoint of each nucleus species is high, say $Z \times 10^{21} \mathrm{eV}$. Then presumably, protons would be the dominant component up to energies close to $10^{21} \mathrm{eV}$, since hydrogen is the most abundant element. The addition of the significant nucleon fraction from photodisintegrated heavy elements would only serve to strengthen the proton dominance. A very recent paper (Ref. [42]) addressed this issue using injected mixtures of iron nuclei and protons for an $E_{\max }$ close to $4 \times 10^{20} \mathrm{eV}$. They found that for these energies a small component of a few percent iron still dominated the spectrum and composition at high energies. 


\section{Conclusions}

We have performed an exhaustive scan in the source evolution factor $m$, the spectral index $\alpha$ and maximum energy $Z E_{\max }$ of the source spectrum and the minimum distance to the sources $z_{\text {min }}$, for sources emitting only protons, or oxygen or iron nuclei and compared the total predicted flux at Earth above $E_{\text {cut }}=1 \times 10^{19} \mathrm{eV}$ with the latest Pierre Auger spectrum. We have also imposed the predicted spectrum not to exceed the observed one at energies below $1 \times 10^{19} \mathrm{eV}$. For an evolution of sources with $m=4$, consistent with evolution of AGN, the spectrum agrees with not only pure proton injection (with $\alpha=2.2$ and $E_{\max }=10^{20.2}-10^{21.1} \mathrm{eV}$ ) but also iron injection with (with $\alpha=1.6-1.7$ and $E_{\max }=10^{20.2}-10^{20.5}$ ) if the sources are not further away than 50 and $25 \mathrm{Mpc}$ respectively.

For smaller $m$, in particular $m=0$, we find solutions with all injected compositions. The iron injection is particularly interesting in that it has two disparate regions of high significance around $Z E_{\max }=26 \times 2 \times 10^{19} \mathrm{eV}$ (with $\alpha=1.7-2.2$ ) and $Z E_{\max }=26 \times 6.4 \times 10^{20} \mathrm{eV}$ (with $\alpha=2.0-2.3$ ) with the intermediate $E_{\max }$ cases much less favorable (only the high energy solution remains if the sources are very far way). Our results for $m=0$ and $z_{\min }$ seem to be in agreement with the results of Ref. [42], which appeared while we were finishing writing the present paper.

We have also studied the effects of $E_{\text {cut }}$ and shown that the regions of parameter space with good fits depends strongly on it. This is easily understood, since there are more events per bin at low energies, thus the error bars are smaller and fewer models provide a good fit for lower $E_{\text {cut }}$. Each $E_{\text {cut }}$ is appropriate for different hypotheses for the energy at which the transition to extra-galactic sources occurs. For $E_{\text {cut }}=2.5 \times 10^{18} \mathrm{eV}$ and pure proton injection, corresponding to the "dip model" of Ref. [16] only $\alpha=2.2$ provide models with a non negligible goodness of fit, with $E_{\max }=10^{20.5} \mathrm{eV}$ providing the best fit (although with $p<0.05$ ). The almost good proton models for this low $E_{\text {cut }}$ have a deficit of flux in the first fitted bin, at the $10^{18.4} \mathrm{eV}$ bin, which has the smallest error bar. So presumably, if an LEC is added to match the flux exactly at that bin, their goodness of fit would improve. Also, if the first bin is eliminated from the fit, the best fit point just mentioned has $p \geq 0.05$. This disagreement of the fit of the "dip model" to the Auger spectrum using surface detector data due to the lowest energy bins, coincides with the recent findings of Berezinky [43] using a different statistical method. For $E_{\text {cut }}=4 \times 10^{19} \mathrm{eV}$, good models are found regardless of $E_{\max }$, but a suitable low energy component should become important up to energies close to $4 \times 10^{19} \mathrm{eV}$.

The spectrum favors a minimum distance to sources, $z_{\text {min }}$, that is as small as possible and the degeneracy between $\alpha$ and $m$ was also demonstrated.

The three models that have the highest probability to describe the observed spectrum paint very different pictures of cosmic ray composition. For the pure proton injection at the source all UHECR primaries should be protons (possible with some GZK photons), while the two iron injection cases lead to a mixed composition separable by a distinctive abundance of UHECR proton primaries. The low $E_{\max }$ Fe injection case 
predicts that $\sim 90 \%$ of the primaries above an energy of $1 \times 10^{19} \mathrm{eV}$ are elements with an atomic weight greater than $30 \mathrm{amu}$, whereas the high $E_{\max }$ case contains a fraction of protons only slightly smaller than the total flux of elements with an atomic weight greater than $30 \mathrm{amu}$. In both cases the average atomic weight would be considered heavy.

If the hint of a transition from light element dominance to heavy element dominance in the composition of UHECR above $2 \times 10^{19} \mathrm{eV}$ seen in the latest results of the Pierre Auger Observatory turns out to be true, then the highest energy cosmic rays are likely to contain a large fraction of heavy elements. Both the low $E_{\max }$ case and the high $E_{\max }$ case present an intriguing scenario for a mixed composition. Pure iron injection at the sources is unlikely, so if cosmic rays are of mixed composition with maximum energy at the source of each nucleus species equal to $Z \times 10^{19} \mathrm{eV}$, then the low $E_{\max }$ case results in a composition that becomes heavier with energy until only iron primaries remain. In the high $E_{\max }$ case the composition also becomes heavier with energy, but should maintain a significant flux of protons well beyond the GZK energy, coming both from the proton injection itself and from the photodisintegration of the heavy elements. This certainly presents an intriguing direction for composition and spectrum studies in the future.

\section{Acknowledgments}

The work of G.G and O.K. was supported in part by NASA grants NAG5-13399 and ATP03-0000-0057. G.G was also supported in part by the US DOE grant DE-FG0391ER40662 Task C. K.A, J.L and M.H were supported in part by US DOE grant DE-FG03-91ER40662 Task F. The numerical part of this work was performed at the computer cluster of the INR RAS Theory Division, the "Neutrino" cluster of the UCLA Physics and Astronomy Department, as well as the Pierre Auger cluster of the UCLA Physics and Astronomy Department.

[1] K. Greisen, Phys. Rev. Lett. 16, 748 (1966). G. T. Zatsepin and V. A. Kuzmin, JETP Lett. 4, 78 (1966) [Pisma Zh. Eksp. Teor. Fiz. 4, 114 (1966)].

[2] M. Takeda et al., Phys. Rev. Lett. 81, 1163 (1998); see N. Hayashida et al., astro-ph/0008102, for an update; see also http ://www-akeno.icrr.u-tokyo.ac.jp/AGASA/.

[3] R. U. Abbasi et al. [High Resolution Fly's Eye Collaboration], Phys. Rev. Lett. 92, 151101 (2004); see also http ://hires.physics.utah.edu/.

[4] R. Abbasi et al. [HiRes Collaboration], astro-ph/0703099.

[5] Pierre Auger Observatory, http://www.auger.org.

[6] M. Roth et al. [Pierre Auger Collaboration], "Measurement of the UHECR energy spectrum using data from the Surface Detector of the Pierre Auger Observatory," Proc. 30 ${ }^{\text {th }}$ ICRC, Mérida (2007).

[7] P. Sommers et al. [Pierre Auger Collaboration], Proc. 29 ${ }^{\text {th }}$ ICRC, Pune (2005) 7, 387.

[8] D. Allard et al. [Pierre Auger Collaboration], Proc. $29^{\text {th }}$ ICRC, Pune (2005) 7, 71.

[9] M. Ave et al. [Pierre Auger Collaboration], "Reconstruction accuracy of the surface detector of the Pierre Auger Observatory," Proc. 30 ${ }^{\text {th }}$ ICRC, Mérida (2007). 
[10] M. Takeda et al., Astropart. Phys. 19, 447 (2003) astro-ph/0209422.

[11] F.W. Stecker, Phys. Lett. 21, 1016 (1968); S. Yoshida and M. Teshima, Prog. Theor. Phys. 89, 833 (1993); F. A. Aharonian and J. W. Cronin, Phys. Rev. D50, 1892 (1994); J. W. Elbert and P. Sommers, Astrophys. J. 441, 151 (1995);

[12] F. Halzen, R. A. Vazquez, T. Stanev, and V. P. Vankov, Astropart. Phys., 3, 151 (1995).

[13] K. Dolag, D. Grasso, V. Springel and I. Tkachev, JETP Lett. 79, 583 (2004) [Pisma Zh. Eksp. Teor. Fiz. 79, 719 (2004)]; and JCAP 0501, 009 (2005).

[14] G. Sigl, F. Miniati and T. A. Ensslin, Phys. Rev. D 68, 043002 (2003); astro-ph/0309695 Phys. Rev. D 70, 043007 (2004); astro-ph/0409098.

[15] G. Hughes et al. [HiRes Collaboration], "A Measurement of the Average Longitudinal Development Profile of Cosmic Ray Air Showers from $10^{17} \mathrm{eV}$ to $10^{20} \mathrm{eV}$," Proc. $30^{\text {th }}$ ICRC, Mérida (2007).

[16] V. Berezinsky, A. Z. Gazizov and S. I. Grigorieva, hep-ph/0204357.

[17] M. Unger et al. [Pierre Auger Collaboration], "Study of the Cosmic Ray Composition above 0.4 EeV using the Longitudinal Profiles of Showers observed at the Pierre Auger Observatory," Proc. $30^{\text {th }}$ ICRC, Mérida (2007).

[18] T. T. Stanev, Astrophys. J. 479, 290 (1997); G. A. Medina-Tanco, E. M. de Gouveia Dal Pino and J. E. Horvath, astro-ph/9707041; M. Prouza and R. Smida, astro-ph/0307165

[19] O.E. Kalashev, V.A. Kuzmin and D.V. Semikoz, astro-ph/9911035. Mod. Phys. Lett. A 16, 2505 (2001); O.E. Kalashev Ph.D. Thesis, INR RAS, 2003.

[20] G. B. Gelmini, O. Kalashev and D. V. Semikoz, arXiv:0706.2181 [astro-ph].

[21] F. W. Stecker, M. A. Malkan and S. T. Scully, Astrophys. J. 648, 774 (2006).

[22] V. S. Berezinsky, et al, "Astrophysics of Cosmic Rays." (North-Holland, Amsterdam, 1990); T.K. Gaisser, "Cosmic Rays and Particle Physics." (Cambridge University Press, Cambridge, England, 1990).

[23] R.J. Protheroe, In "Topics in cosmic-ray astrophysics", ed. M. A. DuVernois, Nova Science Publishing: New York, 1999, astro-ph/9812055); M.A. Malkov, Ap.J. 511, L53 (1999); K. Mannheim, R.J. Protheroe, J. P. Rachen, Phys. Rev. D63, 023003 (2001).

[24] E. V. Derishev, F. A. Aharonian, V. V. Kocharovsky and V. V. Kocharovsky, Phys. Rev. D 68, 043003 (2003).

[25] A. Neronov and D. Semikoz, New Astronomy Reviews 47, 693 (2003); A. Neronov, P. Tinyakov and I. Tkachev, J. Exp. Theor. Phys. 100, 656 (2005) [Zh. Eksp. Teor. Fiz. 100, 744 (2005)] astro-ph/0402132.

[26] V. Berezinsky, A. Gazizov and S. Grigorieva, astro-ph/0210095 Phys. Lett. B 612, 147 (2005).

[27] J. S. Dunlop and J. A. Peacock, MNRAS 247, 19 (1990).

[28] D. De Marco, T. Stanev and F. W. Stecker, Phys. Rev. D 73, 043003 (2006)

[29] S. Inoue, G. Sigl, F. Miniati and E. Armengaud, astro-ph/0701167.

[30] M. Takeda et al., Astrophys. J. 522, 225 (1999).

[31] R. U. Abbasi et al. [HIRES], Astrophys. J. 610, L73 (2004). astro-ph/0404137.

[32] R. U. Abbasi et al. [The High Resolution Fly's Eye Collaboration (HIRES)], astro-ph/0412617.

[33] H. Yoshiguchi, S. Nagataki and K. Sato, Astrophys. J. 614, 43 (2004); M. Kachelriess and D. Semikoz, Astropart. Phys. 23, 486 (2005).

[34] E. Waxman, K. B. Fisher and T. Piran, Astrophys. J. 483, 1 (1997); S. L. Dubovsky, P. G. Tinyakov and I. I. Tkachev, Phys. Rev. Lett. 85, 1154 (2000); Z. Fodor and S. D. Katz, Phys. Rev. D 63, 023002 (2001); H. Yoshiguchi, S. Nagataki, S. Tsubaki and K. Sato, Astrophys. J. 586, 1211 (2003) [Erratum-ibid. 601, 592 (2004)]; H. Yoshiguchi, S. Nagataki and K. Sato, Astrophys. J. 592, 311 (2003); P. Blasi and D. De Marco, Astropart. Phys. 20, 559 (2004).

[35] P. Sreekumar et al. [EGRET Collaboration], Astrophys. J. 494, 523 (1998).

[36] G. Gelmini, O. Kalashev and D. V. Semikoz, astro-ph/0702464.

[37] I. F. M. Albuquerque and G. F. Smoot, Astropart. Phys. 25, 375 (2006).

[38] J. Abraham et al. [Pierre Auger Collaboration], "Upper Limit on the Cosmic Ray Photon Flux Above $10^{19} \mathrm{eV}$ Using the Surface Detector of the Pierre Auger Observatory," Submitted to 
Astrop. Phys. (2007).

[39] G. Gelmini, O. Kalashev and D. V. Semikoz, arXiv:astro-ph/0506128, version 3 of 10/1/07.

[40] Z. Fodor, S. D. Katz and A. Ringwald, Phys. Rev. Lett. 88, 171101 (2002) and JHEP 0206, 046 (2002).

[41] S. Baker and R.D. Cousins, Nucl. Instrum. Methods 221, 437 (1984); Particle Data Group's Statistics Review (2004).

[42] L. A. Anchordoqui, H. Goldberg, D. Hooper, S. Sarkar and A. M. Taylor, arXiv:0709.0734 [astro$\mathrm{ph}$.

[43] V. Berezinsky, arXiv:0710.2750 [astro-ph]. 